\title{
An Analysis of SAT-Based Model Checking Techniques in an Industrial Environment
}

\author{
Nina Amla, Xiaoqun Du, Andreas Kuehlmann, Robert P. Kurshan, \\ and Kenneth L. McMillan \\ Cadence Design Systems
}

\begin{abstract}
Model checking is a formal technique for automatically verifying that a finite-state model satisfies a temporal property. In model checking, generally Binary Decision Diagrams (BDDs) are used to efficiently encode the transition relation of the finite-state model. Recently model checking algorithms based on Boolean satisfiability (SAT) procedures have been developed to complement the traditional BDD-based model checking. These algorithms can be broadly classified into three categories: (1) bounded model checking which is useful for finding failures (2) hybrid algorithms that combine SAT and BDD based methods for unbounded model checking, and (3) purely SAT-based unbounded model checking algorithms. The goal of this paper is to provide a uniform and comprehensive basis for evaluating these algorithms. The paper describes eight bounded and unbounded techniques, and analyzes the performance of these algorithms on a large and diverse set of hardware benchmarks.
\end{abstract}

\section{Introduction}

A common method used in formal verification is model checking [7]26]. Generally, Binary Decision Diagrams (BDDs) 4] are used to symbolically represent the set of states. This approach, known as symbolic model checking [5], has been successfully applied in practice. Unfortunately, BDDs are very sensitive to the type and size of the system. For instance common designs like multipliers can not be represented efficiently with BDDs. Due to recent advances in tools [19]2311] that solve the Boolean satisfiability problem (SAT), formal reasoning based on SAT is proving to be an viable alternative to BDDs.

Bounded Model Checking (BMC) [3] is a SAT-based technique where a system is unfolded $k$ times and encoded as a SAT problem to be solved by a CNF-based SAT solver. A satisfying assignment returned by the SAT solver corresponds to a counterexample of length $k$. If the problem is determined to be unsatisfiable, the SAT solver produces a proof of the fact that there are no counterexamples of length $k$. A different approach, called circuit-based BMC [15], uses the circuit structure to make BMC more efficient. The circuit is unfolded incrementally and at each step equivalent nodes are identified and merged to simplify the circuit. BMC, while successful in finding errors, is incomplete: there is no efficient way to decide that the property is true. Recently several complete model checking algorithms have been developed that use SAT-based quantifier 
elimination 2010], ATPG methods [12], and combinations of SAT-based BMC with techniques like BDD-based model checking [6]22, induction [27] and interpolation [21].

Since users have limited resources for the verification of systems, it is important to know which of these new SAT-based algorithms is most effective. This paper presents an experimental analysis of these bounded and unbounded algorithms in an attempt to address this issue. Unlike previous efforts that compared SAT-based BMC to BDD-based and explicit state methods (cf. [81]), this paper focuses only on SAT-based techniques. In Section 2 we give an overview of the eight algorithms we evaluated. A more comprehensive survey of SAT-based techniques can be found in 25. We describe our experimental framework in Section 3. We compare the various algorithms on a set of over 1000 examples drawn from actual hardware designs. Section 4 presents our results and analysis. We conclude and discuss future work in Section 5 .

\section{Overview of the Algorithms}

\subsection{Preliminaries}

A model $M=(S, I, T, L)$ has a set of states $S$, a set of initial states $I \subseteq S$, a transition relation $T \subseteq S \times S$, and a labeling function $L: S \rightarrow 2^{A}$ where $A$ is a set of atomic propositions. For the purposes of this paper, we shall consider only invariant properties specified in the logic LTL. The construction given in 16 can be used to reduce model checking of safety properties to checking invariant properties. The syntax and semantics of LTL and other temporal logics is not given here but can be found in 9 .

Given a finite state model $M$ and a safety property $p$, the model checking algorithm checks that $M$ satisfies $p$, written $M \models p$. The forward reachability algorithm starts at the initial states and computes the image, which is the set of states reachable in one step. This procedure is continued until either the property is falsified in some state or no new states are encountered (a fixed point). The backward reachability algorithm works similarly but starts from the states where the property is false and computes the preimage, which is the set of states that can reach the current states in one step. The representation and manipulation of the sets of states can be done explicitly or with Binary Decision Diagrams (BDDs). In the sequel, we shall refer to BDD-based model checking as MC.

\subsection{DPLL-Style SAT Solvers}

The Boolean satisfiability problem (SAT) is to determine if a given Boolean formula has a satisfying assignment. This is generally done by converting the formula into Conjunctive Normal Form (CNF), which can be efficiently solved by a SAT solver. A key operation used in SAT solvers is resolution, where two clauses $(a \vee b)$ and $(\neg a \vee c)$ can be resolved to give a new clause $(b \vee c)$. Modern 
DPLL-style SAT solvers [192311] make assignments to variables, called decisions, and generate an implication graph which records the decisions and the effects of Boolean constraint propagation. When all the variables are assigned, the SAT solver terminates with the satisfying assignment. But if there is a conflict, which is a clause where the negation of every literal already appears in the implication graph, a conflict clause is generated through resolution. This conflict clause is added to the formula to avoid making those assignments again. The SAT solver then backtracks to undo some of the conflicting assignments. The SAT solver terminates with an unsatisfiable answer when it rules out all possible assignments. The resolution steps used in generating the conflict clauses can now be used to produce a proof of unsatisfiability.

\subsection{SAT-Based Bounded Model Checking}

Bounded Model Checking (BMC) [3] is a restricted form of model checking, where one searches for a counterexample (CEX) in executions bounded by some length $k$. In this approach the model is unfolded $k$ times, conjuncted with the negation of the property, and then encoded as a propositional satisfiability formula. Given a model $M$ and an invariant property $p$, the BMC problem is encoded as follows:

$$
B M C(M, p, k)=I\left(s_{0}\right) \wedge \bigwedge_{i=0}^{k-1} T\left(s_{i}, s_{i+1}\right) \wedge \bigvee_{i=0}^{k} \neg p\left(s_{i}\right)
$$

The formula can be converted into CNF and solved by a SAT solver. If the formula is satisfiable, then the property is false, and the SAT solver has found a satisfying assignment that corresponds to a counterexample of length $k$. In the unsatisfiable case, there is no counterexample of length $k$ and a proof of unsatisfiability can be obtained from the SAT solver.

\subsection{Circuit-Based Bounded Model Checking}

In circuit-based BMC the circuit structure is exploited to enhance efficiency. Rather than translating the problem into a CNF formula directly, circuit-based $\mathrm{BMC}$ uses an intermediate representation, called And-Inverter Graphs (AIGs) 15, that keeps the circuit structure. The use of AIGs allows the application of the SAT-sweeping technique [14, where one identifies equivalent nodes using a SAT solver and merges these equivalent nodes to simplify the circuit represented by the AIG. Random simulation is used to pick candidate pairs of nodes that have identical simulation results, and a SAT solver is used to check whether the XOR of the two candidate nodes can ever be satisfied. If not, the nodes are equivalent and can be merged to simplify the AIG. If the XOR of the nodes is satisfiable, the SAT solver will give a witness that shows how the nodes can obtain different values. This witness can be used to show the in-equivalence of other nodes to reduce the number of candidate pairs for equivalence-finding. After the completion of SAT-sweeping, the simplified AIG is translated into a CNF formula for BMC. 


\subsection{CEX-Based Abstraction Refinement}

Counterexample-based abstraction-refinement [17] is an iterative technique that starts with BDD-based MC on an initial conservative abstraction of the model. If $\mathrm{MC}$ proves the property on the abstraction then the property is true on the full model. However, if a counterexample $A$ is found, it could either be an actual error or it may be spurious, in which case one needs to refine the abstraction to rule out this counterexample. The process is then repeated until the property is found to be true, or until a real counterexample is produced.

The counterexample-based method in [6] used BMC to concretize the counterexample by solving the following:

$$
B M C(M, p, k, A)=I\left(s_{0}\right) \wedge \bigwedge_{i=0}^{k-1} T\left(s_{i}, s_{i+1}\right) \wedge \bigvee_{i=0}^{k} \neg p\left(s_{i}\right) \wedge \bigwedge_{i=0}^{k} A_{i}
$$

where $A_{i}$ is a constraint that represents the assignments in the abstract counterexample $A$ in time frame $i$. If this formula is determined to be satisfiable then the satisfying assignment represents a counterexample on the concrete model. In the unsatisfiable case, the method 6] analyzes the proof of unsatisfiability generated by the SAT solver to find a set of constraints whose addition to the abstraction will rule out this spurious counterexample. Since the BMC problem includes the constraints in the abstract counterexample $A$, one can guarantee that $A$ is eliminated by adding all variables that occur in the proof to the existing abstraction. The pseudocode is shown in Figure 1.

procedure cex-based $(M, p)$

1. generate initial abstraction $M^{\prime}$

2. while true do

3. if $M C\left(M^{\prime}, p\right)$ holds then return verified

4. let $k=$ length of abstract counterexample $A$

5. if $B M C(M, p, k, A)$ is SAT then return counterexample

6. $\quad$ else use proof of UNSAT $P$ to refine $M^{\prime}$

7. end while

end

Fig. 1. SAT-based counterexample procedure

\subsection{Proof-Based Abstraction Refinement}

The proof-based algorithm in [22] also iterates through SAT-based BMC and BDD-based MC. It starts with a short BMC run, and if the problem is satisfiable, an error has been found. If the problem is unsatisfiable, the proof of unsatisfiability is used to guide the formation of a new conservative abstraction on which BDD-based MC is run. In the case that the BDD-based model checker proves the property then the algorithm terminates; otherwise the length $k^{\prime}$ of the counterexample generated by the model checker is used as the next 
procedure proof-based $(M, p)$

1. initialize $k$

2. while true do

3. if $B M C(M, p, k)$ is SAT then return counterexample

4. else

5. derive new abstraction $M^{\prime}$ from proof $P$

6. $\quad$ if $M C\left(M^{\prime}, p\right)$ holds then return verified

7. $\quad$ else set $k$ to length of counterexample $k^{\prime}$

8. end while

end

Fig. 2. Proof-based procedure

BMC length. Notice that only the length of the counterexample generated by the BDD-based MC is used. This method creates a new abstraction in each iteration, in contrast to the counterexample method which refines the existing abstraction. Since this abstraction includes all the variables in the proof of unsatisfiability for a BMC run up to depth $k$, we know that any counterexample obtained from model checking this abstract model will be of length greater than $k$. Therefore, unlike the counterexample method, this algorithm eliminates all counterexamples of length $k$ in a single unsatisfiable BMC run. This procedure, shown in Figure 2, is continued until either a failure is found in the BMC phase or the property is proved in the BDD-based MC phase. The termination of the algorithm hinges on the fact that the value of $k^{\prime}$ increases in every iteration.

\subsection{Induction-Based Model Checking}

The induction-based method in [27] uses a SAT solver as the decision procedure for a special kind of induction called $k$-induction. In this type of induction, one attempts to prove that a property holds in the current state, assuming that it holds in the previous $k$ consecutive states. In addition, for completeness, one has to add an additional constraint that specifies that the states along a path must be unique. This is formalized as follows:

$$
\begin{array}{r}
\operatorname{Base}(M, p, k)=I\left(s_{0}\right) \wedge \bigwedge_{i=0}^{k-1} T\left(s_{i}, s_{i+1}\right) \wedge \bigvee_{i=0}^{k} \neg p\left(s_{i}\right) \\
\operatorname{Step}(M, p, k)=\bigwedge_{0 \leq i<j \leq k} s_{i} \neq s_{j} \wedge \bigwedge_{i=0}^{k} T\left(s_{i}, s_{i+1}\right) \wedge \bigwedge_{i=0}^{k} p\left(s_{i}\right) \wedge \neg p\left(s_{k+1}\right)
\end{array}
$$

A counterexample has been found if the base condition is satisfiable; otherwise the value of $k$ is increased until both conditions are unsatisfiable, which means the property holds. The pseudocode is shown in Figure 3 . 
procedure $k$-induction $(M, p)$

1. initialize $k=0$

2. while true do

3. if $\operatorname{Base}(M, p, k)$ is $\mathrm{SAT}$ then return counterexample

4. else if $\operatorname{Step}(M, p, k)$ is $\operatorname{UNSAT}$ then return verified

5. $k=k+1$

6. end while

end

Fig. 3. The $k$-induction procedure

\subsection{Interpolation-Based Model Checking}

An interpolant $\mathcal{I}$ for an unsatisfiable formula $A \wedge B$ is a formula such that: (1) $A \Rightarrow \mathcal{I}(2) \mathcal{I} \wedge B$ is unsatisfiable and (3) $\mathcal{I}$ refers only to the common variables of $A$ and $B$. Intuitively, $\mathcal{I}$ is the set of facts that the SAT solver considers relevant in proving the unsatisfiability of $A \wedge B$.

The interpolation-based algorithm [21] uses interpolants to derive an overapproximation of the reachable states with respect to the property. This is done as follows (Figure 4). The BMC problem $B M C(M, p, k)$ is solved for an initial depth $k$. If the problem is satisfiable, a counterexample is returned, and the algorithm terminates. If $\operatorname{BMC}(M, p, k)$ is unsatisfiable, the formula representing the problem is partitioned into $\operatorname{Pref}(M, p, k) \wedge S u f f(M, p, k)$, where $\operatorname{Pref}(M, p, k)$ is the conjunction of the initial condition and the first transition, and $\operatorname{Suff}(M, p, k)$ is the conjunction of the rest of the transitions and the final condition. The interpolant $\mathcal{I}$ of $\operatorname{Pref}(M, p, k)$ and $\operatorname{Suff}(M, p, k)$ is computed. Since $\operatorname{Pref}(M, p, k) \Rightarrow \mathcal{I}$, it follows that $\mathcal{I}$ is true in all states reachable from $I\left(s_{0}\right)$ in one step. This means that $\mathcal{I}$ is an over-approximation of the set of states reachable from $I\left(s_{0}\right)$ in one step. Also, since $\mathcal{I} \wedge S u f f(M, p, k)$ is unsatisfiable, it also follows that no state satisfying $\mathcal{I}$ can reach an error in $k-1$ steps. If $\mathcal{I}$ contains no new states, that is, $\mathcal{I} \Rightarrow I\left(s_{0}\right)$, then a fixed point of the reachable set of states has been reached, thus the property holds. If $\mathcal{I}$ has new states then $R^{\prime}$ represents an over-approximation of the states reached so far. The algorithm then uses $R^{\prime}$ to replace the initial set $I$, and iterates the process of solving the BMC problem at depth $k$ and generating the interpolant as the over-approximation of the set of states reachable in the next step. The property is determined to be true when the BMC problem with $R^{\prime}$ as the initial condition is unsatisfiable, and its interpolant leads to a fixed point of reachable states. However, if the BMC problem is satisfiable, the counterexample may be spurious since $R^{\prime}$ is an over-approximation of the reachable set of states. In this case, the value of $k$ is increased, and the procedure is continued. The algorithm will eventually terminate when $k$ becomes larger than the diameter of the model.

\subsection{Quantification-Based Model Checking}

There are many approaches [25] to doing quantifier elimination which is a key step in reachability analysis. The purely SAT-based quantifier elimination procedure introduced in [20] works by enumeration of all the satisfying assignments. 
procedure interpolation $(M, p)$

1. initialize $k$

2. while true do

3. if $B M C(M, p, k)$ is SAT then return counterexample

4. $R=I$

5. while true do

6. $\quad M^{\prime}=(S, R, T, L)$

7. $\quad$ let $C=\operatorname{Pref}\left(M^{\prime}, p, k\right) \wedge \operatorname{Suff}\left(M^{\prime}, p, k\right)$

8. if $C$ is SAT then break (goto line 15)

9. $\quad / * C$ is $\mathrm{UNSAT}^{*} /$

10. compute interpolant $\mathcal{I}$ of $\operatorname{Pref}\left(M^{\prime}, p, k\right) \wedge \operatorname{Suff}\left(M^{\prime}, p, k\right)$

11. $R^{\prime}=\mathcal{I}$ is an over-approximation of states reachable from $R$ in one step.

12. $\quad$ if $R \Rightarrow R^{\prime}$ then return verified

13. $R=R \vee R^{\prime}$

14. end while

15. increase $k$

16. end while

end

Fig. 4. Interpolation procedure

The SAT solver is modified to generate all the satisfying assignments by adding blocking clauses to the problem each time an assignment is found. The SAT solving process is continued until no new solutions are found. A blocking clause, which refers only to the state variables, represents the negation of a state cube. This quantification procedure yields a purely SAT-based method for computing the preimage in backward symbolic model checking.

A recent quantification-based method [10] uses a circuit representation of the blocking constraints and use a hybrid solver that works directly on this representation. This enables circuit cofactoring with respect to the input assignments to simplify the circuit graph in each enumeration step. This results in more solutions in each enumeration step and thus far fewer enumerations steps. It is reported in [10] that this method outperforms the technique described in [20. We implemented the basic cofactoring-based quantification approach in our framework. Our implementation did not include the heuristics provided in [10] to select values for unassigned inputs in the satisfying cube; we just use the complete input assignment provided by the SAT solver in that enumeration step. We also did not use functional hashing in the simplification process but we did use structural hashing.

\subsection{ATPG-Based Model Checking}

Automatic Test Pattern Generation (ATPG) is an approach that adapts DPLLstyle SAT techniques to a structural representation of a circuit. The ATPG-based algorithm in 12 combines the structure guided search strategy of ATPG with the faster implication procedures and conflict-based learning in SAT solvers. They use a circuit representation, a CNF clause database and a mapping between both representations. The method conducts a backward search, using an ATPG- 
based back-tracing traversal method, from the states where the property is false. The search strategy, which is a mixture of DFS and BFS, is based on a cost function that measures the number of states traversed. A counterexample is generated if an initial state is reached during the search; otherwise the property is proved to be true if the entire backward reachable set of states does not intersect with the initial state set. This procedure is complete because the search is efficiently bounded by using additional Boolean constraints to mark visited states as already reached and hence never to be visited again.

\section{Experimental Framework}

In order to measure the relative performance of the algorithms described in the previous section, we implemented all the methods except the ATPG-based method SATORI, which was developed at University of Santa Barbara [12. We developed a flexible experimental framework that allows external tools, like SATORI, to be integrated with little effort. We use a simple intermediate representation that can be translated easily and efficiently into the input language of various tools. This interface also enables us to plug and play with different SAT solvers and BDD packages.

\subsection{Benchmarks}

In the context of commercial software development, a good benchmark suite must be large, diverse, and representative of real customer designs. The data collection must be fully automated, and must complete within a reasonable amount of time so that the benchmark suite can be used as a regression suite for tracking the performance of the software over time.

Our benchmark suite included approximately 85 hardware designs, accumulated through many years of customer interaction. The sizes of these designs ranged from a few hundred to more than 100,000 lines of HDL code. Each design in our benchmark suite contained from one up to a few hundred properties to check. Some of the properties were duplicates because they were instantiated from the same property declaration in similar parts of a design. To make our benchmark suite as diverse as possible, we removed all duplicate properties, where two properties were considered duplicates if the model had the same number of state and combinational variables, and that the running times were within $10 \%$ of each other.

There were properties that none of the algorithms could finish within a reasonable amount of time. We removed most of these properties from our benchmark suite because they were not useful for comparing the relative performance of the algorithms. We did keep some of these properties to track performance improvements of the algorithms over time. This resulted in a total of 1182 properties for the 85 designs in our benchmark suite. Out of these 1182 properties, 803 of them are passes, 364 of them are failures, and the remaining 15 properties have unknown results. 


\subsection{Data Collection}

Each 〈property, algorithm〉 pair corresponds to one run for data collection. This meant we needed 1182 runs for each technique, hence it was important that we set up our test environment so that the experiments finished within a reasonable amount of time. To do this, we set a time limit of 3600 seconds for each property. We found in our experiments that a majority of the runs finished within this time limit.

We used a computer server farm for data collection. In our experiments, we use 10 identical Redhat Enterprise Linux machines, each with an AMD Opteron $\mathrm{CPU}$ at $2 \mathrm{GHZ}$ and $4 \mathrm{~GB}$ of available memory. We partitioned our entire set of runs into multiple jobs, each job consisting of a small set of runs. These jobs are submitted to the server farm and launched whenever a CPU is free. To ensure the accurate collection of data, no other jobs are permitted on a CPU when it is running one of our data collection jobs; also, a data collection job cannot be started unless a machine has at least $4 \mathrm{~GB}$ of free memory.

\section{Results and Analysis}

In our experiments, except for SATORI, we used the same SAT solver and BDDbased model checker for all the techniques. The SAT solver is incremental [29], in the sense that it is possible to add/delete clauses and restart the solver, while maintaining all previously inferred conflict clauses that were not derived from deleted clauses. An important point to note is that all methods were run with default settings and there was no tuning done with respect to specific examples.

Table 1. Summary Table for the Bounded Technique

\begin{tabular}{|c|c|c|c|c|c|}
\hline Depth & \# Props & $\begin{array}{r}\mathrm{SA}^{\prime} \\
\# \text { Fin }\end{array}$ & $\begin{array}{l}\text { T-BMC } \\
\text { Avg Time }\end{array}$ & $\begin{array}{r}\text { CII } \\
\# \text { Fin }\end{array}$ & $\begin{array}{l}\text { R-BMC } \\
\text { Avg Time }\end{array}$ \\
\hline 10 & $\overline{1182}$ & $\overline{1179}$ & 10.9 & 1178 & 15.0 \\
\hline 25 & 1182 & 1175 & 28.8 & 1177 & 23.8 \\
\hline 50 & 1182 & 1168 & 73.3 & 1170 & 53.3 \\
\hline 100 & 1182 & 1153 & 174.0 & 1158 & 117.0 \\
\hline
\end{tabular}

For the bounded model checking techniques, we set a time limit of $3600 \mathrm{sec}-$ onds and did four runs with depth limits of 10, 25, 50 and 100. We measured the number of problems that were resolved within the time limit and the average time taken per property (over all the properties regardless of whether an algorithm finished or not) by both methods. Table 1 presents these results. We can see that, after depth 25, the circuit-based approach takes less time on average. We plot the run time at depth 100 for both algorithms in Figure 5, a point below the diagonal line indicates that circuit BMC was faster on that example. In all the tables and plots, the time for any unresolved property is taken to be 
3600 seconds even if the method ran out of memory in far less time. The data shows that the savings due to SAT sweeping in circuit-based BMC outweighs the overhead at the larger depths.
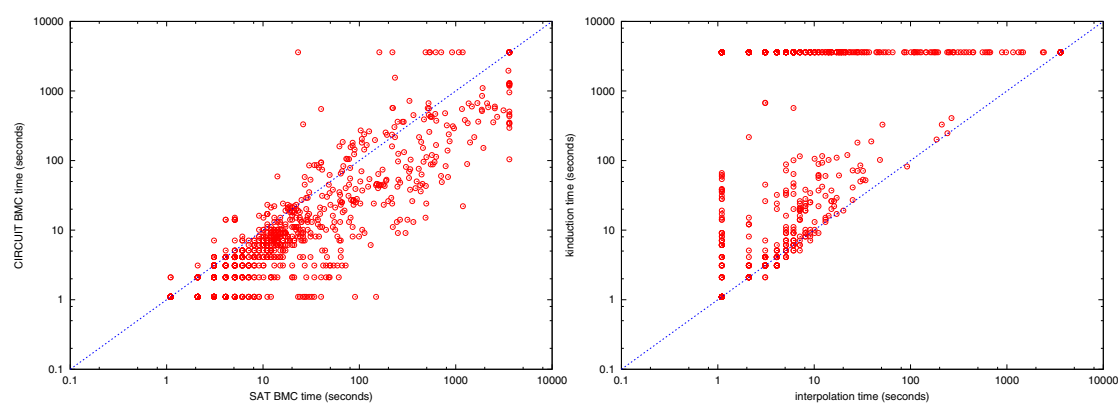

Fig. 5. Plot of time in seconds. Left: $\mathrm{X}$-axis is SAT BMC and Y-axis is Circuit BMC at depth 100. Right: X-axis is Interpolation and Y-axis is K-induction.

For the unbounded techniques, we set a time limit of 3600 seconds for verification, and measure the number of problems that were resolved within this time limit. Table 2 reports the number of resolved problems and average time taken per property. As a baseline, we include the results for a forward traversal BDD-based MC method in Table 2. It is interesting to note that all the SATbased algorithms, except the $k$-induction method, do better than BDD-based model checking with respect to the number of problems resolved and average time taken. However, we shall not include BDD-based MC in any further discussions since it is not in the scope of this paper. We also see that the interpolation method resolved more problems and had a lower average running time than the other techniques. Since the interpolation method is the most robust, in the sense that it resolves the largest number of problems, we plotted the run time of the other five unbounded algorithms versus the interpolation algorithm. These are shown in Figures 5 to 7 . The plots indicate that in general the interpolation method is faster and more robust than the other methods, however there are still many cases where the other techniques do better.

Tables 3 and 4 present the number of problems resolved, average time, average final depth and average number of state variables (size), for only the resolved failing and passing problems respectively. The depth information was not available for the ATPG-based method and is therefore excluded from both tables. We also report the number of "wins" with respect to time, where a win is attributed to a particular algorithm if it does better than all others with respect to running time. In the case of a tie, which we defined to be two runs where the difference was less than $5 \%$ of the run time, we award a win for both methods.

The failing properties in our benchmark suite can be roughly characterized with respect to depth as follows: $91 \%$ failed at a depth of 25 or less with $24 \%$ of the failures at a depth of $2,7 \%$ failed between a depth of 26 till 100 and $2 \%$ failed 
Table 2. Summary Table for the Unbounded Techniques

\begin{tabular}{||l|c|l||l|l||}
\hline Algorithm & \# Props & \# Resolved & Total time & Avg. Time \\
\hline \hline BDD & 1182 & 876 & 1171716 & 991.3 \\
\hline \hline proof-based & 1182 & 1121 & 269377 & 227.9 \\
\hline cex-based & 1182 & 1054 & 520570 & 439.3 \\
\hline \hline cofactor & 1182 & 874 & 1154459 & 976.7 \\
\hline atpg-based & 1182 & 992 & 756480 & 640.0 \\
\hline kinduction & 1182 & 513 & 2417662 & 2045.4 \\
\hline interpolation & 1182 & 1157 & 118791 & 100.5 \\
\hline
\end{tabular}
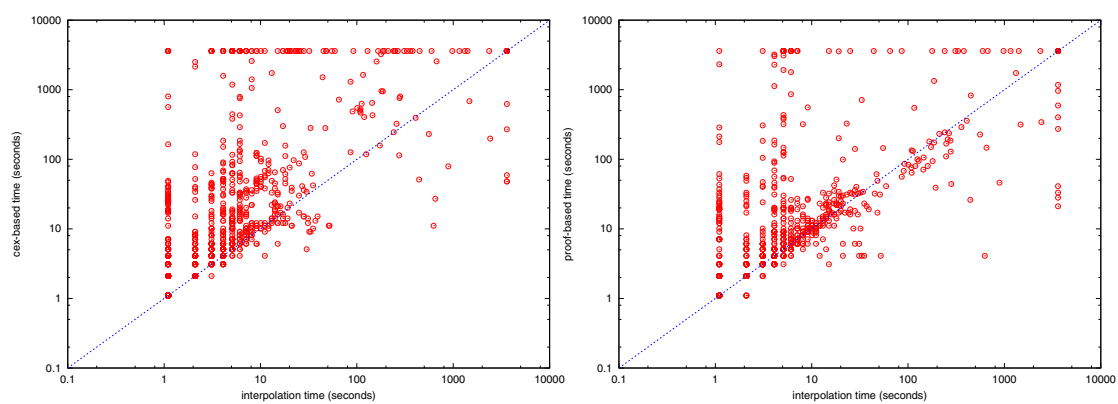

Fig. 6. Plot of time in seconds. Left: $\mathrm{X}$-axis is Interpolation and $\mathrm{Y}$-axis is CEX-based. Right: $\mathrm{X}$-axis is Interpolation and Y-axis is Proof-based.

at a depth greater than 100. The data in Table 3 for the bounded techniques is cumulative, in the sense that we report the total running time at depths 10 , 25 and 50 for an error found at depth 45. Since the maximum depth checked was 100 , the bounded techniques were not able to find failures that occurred at depths greater than 100 and this is reflected in the number of failures. Table 3 shows some interesting trends for the failing properties. Not surprisingly we see that, with respect to average run time, both bounded techniques do better than all others on the failing properties. However, since the bounded techniques were employed at fixed depths, this made finding the shallow errors, like the failures at depth 2, more expensive than necessary. The interpolation and proofbased techniques are competitive with the bounded techniques in number of wins but the proof-based technique is clearly the faster of the two. The $k$-induction method is effective in finding the shallow failures, as is evident in the low run time when it does resolve a problem. The correspondingly low depth numbers in Table 3 are due to the fact that the $k$-induction method ran out of memory fairly early in 662 cases. The mixed DFS/BFS search strategy of the ATPGbased method could cause the technique to miss errors if it chooses to do DFS early and may explain why it does poorly on failures. This is consistent with the results reported in 24. which show that a purely BFS search is more robust than a purely DFS search on failing properties. Another possibility is that, on 
Table 3. Summary Table for the Failing Properties

\begin{tabular}{|c|c|c|c|c|c|}
\hline Algorithm & \# Props & \# Wins & $\begin{array}{r}\text { Failures } \\
\text { Avg Time }\end{array}$ & Avg Size & Avg Depth \\
\hline sat-bmc & 351 & 230 & 9.6 & 106 & 15 \\
\hline circuit-bmc & 350 & 219 & 14.1 & 106 & 15 \\
\hline proof-based & 359 & 216 & $\overline{22.1}$ & 111 & 19 \\
\hline cex-based & 341 & 119 & 58.6 & 88 & 17 \\
\hline cofactor & 268 & 157 & 71.8 & 60 & 18 \\
\hline atpg-based & 295 & 144 & 119.6 & 54 & - \\
\hline kinduction & 340 & 171 & 17.6 & 97 & 7 \\
\hline interpolation & 362 & 224 & 31.6 & 112 & 16 \\
\hline
\end{tabular}

Table 4. Summary Table for the Passing Properties

\begin{tabular}{||l||c|c|c|c|c||}
\hline Algorithm & \multicolumn{5}{c||}{ Passes } \\
& \# Props & \# Wins & Avg Time & Avg Size & Avg Depth \\
\hline \hline proof-based & 762 & 380 & 54.9 & 115 & 30 \\
\hline cex-based & 713 & 237 & 51.7 & 101 & 23 \\
\hline \hline cofactor & 606 & 457 & 48.0 & 109 & 7 \\
\hline atpg-based & 697 & 427 & 53.4 & 111 & - \\
\hline kinduction & 173 & 107 & 19.1 & 14 & 14 \\
\hline interpolation & 795 & 701 & 21.9 & 130 & 22 \\
\hline
\end{tabular}

these examples, the set of states grows faster with backward exploration than with forward exploration. This could in part explain why the cofactoring method does poorly as well. Both the ATPG and cofactoring methods have a much lower average size which suggests that these methods are unable to resolve the larger examples.

For the passing properties, the interpolation technique is the fastest and solves more properties than the other methods. The proof-based technique is the closest in terms of the number of properties resolved but is significantly slower on average. We see that the proof-based method does better than the counterexample-based method, despite the fact that the counterexample-based method proves properties at lower depths on average. This is largely due to the number of iterations done by the counterexample-based method, most of them done refuting counterexamples at the same depth (see [2] for a detailed analysis). The data in Table 4 indicates that the interpolation method is able to prove the properties at a lower depth than the proof-based method. This suggests that the approximate image computation is more effective on these examples than the corresponding BDD-based MC phase in the proof-based method. The $k$-induction method does rather poorly since checking the $k$-induction step is expensive as the value of $k$ gets larger. As mentioned earlier, the size of the BMC problem for the step case is often too large causing the SAT-solver to run out of memory. As 
reported in [18, removing the simple path constraints and trading completeness for efficiency may improve the performance of this method. The ATPG-based and cofactoring methods have a high number of wins and are comparable to the proof-based method in running time. Both methods do backward reachability and cube enlargement but, while their performance signatures are similar, the ATPG method appears to be more robust. The cofactoring method has a low average depth which seems to suggest that a large and rapidly growing backward reachable state space could be contributing to the difference. The search strategy of ATPG-based method permits on-the-fly pruning of the search space, which could be beneficial in such situations. However, we do not have enough data on the ATPG method to validate this conjecture. Furthermore, as observed in [10, using the heuristics to enlarge the satisfying state set in the cofactoring technique has a significant impact on performance.
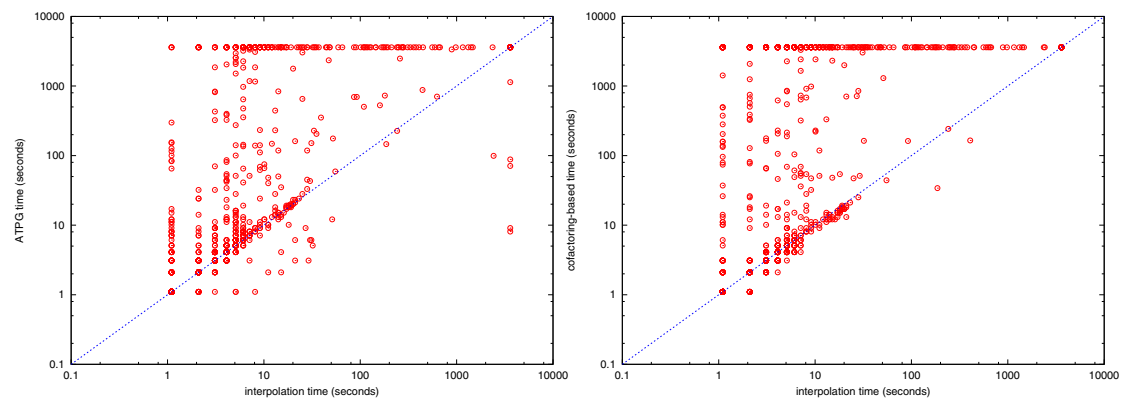

Fig. 7. Plot of time in seconds. Left: X-axis is Interpolation and Y-axis is ATPG-based. Right: $\mathrm{X}$-axis is Interpolation and $\mathrm{Y}$-axis is Cofactoring-based.

\section{Conclusions and Future Work}

This paper compares eight bounded and unbounded SAT-based algorithms on a large set of industrial benchmarks. Our experiments show that although the interpolation technique is the most efficient and robust overall, there were still many examples where the other techniques did better. This is evident in the number of wins in Tables 3 and 4 . Therefore, it would be useful to find ways to apply the best algorithm for each task. One way to do this is to run the algorithms in parallel and terminate the slower ones as soon as the first finishes. Another approach would be to combine the various algorithms in a way that exploits their strengths, like the hybrid method in [2] that combines the proofbased and counterexample-based methods.

For future work, we plan to integrate the VIS model checker into our experimental framework. We believe that methods implemented in VIS would provide some interesting comparisons. The conjecture that the simple path restriction in $k$-induction hinders performance could be evaluated by using the more sophisticated technique described in [18. Furthermore, we could compare the 
counterexample-based technique in 28] that uses a generalized counterexample that is derived from the sequence of reachable states approximations computed by the model checker. Finally it would be useful to evaluate the circuit-based BMC solver described in [13] which uses BDDs to help in the solution of SAT instances given in CNF.

Acknowledgments. The authors thank Kwang-Ting Cheng, Feng Lu, Ganapathy Parthasarthy and Madhu Iyer for help in integrating SATORI.

\section{References}

1. N. Amla, R. Kurshan, K. McMillan, and R. Medel. Experimental analysis of different techniques for bounded model checking. In TACAS, 2003.

2. N. Amla and K. McMillan. A hybrid of counterexample-based and proof-based abstraction. In FMCAD, 2004.

3. A. Biere, A. Cimatti, E. Clarke, and Y. Zhu. Symbolic model checking without BDDs. In TACAS, 1999.

4. R. E. Bryant. Graph-based algorithms for boolean function manipulations. IEEE Transactions on Computers, 1986.

5. J. R. Burch, E. M. Clarke, K. L. McMillan, D.L. Dill, and J. Hwang. Symbolic model checking: $10^{20}$ states and beyond. In LICS, 1990.

6. P. Chauhan, E. Clarke, J. Kukula, S. Sapra, H. Veith, and D. Wang. Automated abstraction refinement for model checking large state spaces using sat based conflict analysis. In FMCAD, 2002.

7. E.M. Clarke and E. A. Emerson. Design and synthesis of synchronization skeletons using branching time temporal logic. In Workshop on Logics of Programs, 1981.

8. F. Copty, L. Fix, R. Fraer, E. Giunchiglia, G. Kamhi, A. Tacchella, and M. Vardi. Benefits of bounded model checking at an industrial setting. In $C A V, 2001$.

9. E. A. Emerson. Temporal and modal logic. In Handbook of Theoretical Computer Science, Volume B: Formal Models and Sematics, 1990.

10. M. Ganai, A. Gupta, and P. Ashar. Efficient SAT-based unbounded symbolic model checking using circuit cofactoring. In ICCAD, 2004.

11. E. Goldberg and Y. Novikov. Berkmin: A fast and robust sat-solver. In DATE, 2002.

12. M. Iyer, G. Parthasarathy, and K.T. Cheng. SATORI- an efficient sequential SAT solver for circuits. In ICCAD, 2003.

13. H. Jin and F. Somenzi. CirCUs: Hybrid satisfiability solver. In SAT, 2004.

14. A. Kuehlmann. Dynamic transition relation simplification for bounded property checking. In ICCAD, 2004.

15. A. Kuehlmann, V. Paruthi, F. Krohm, and M. Ganai. Robust Boolean reasoning for equivalence checking and functional property verification. In TCAD, 2003.

16. O. Kupferman and M. Vardi. Model checking of safety properties. In Formal Methods in System Design, 2001.

17. R. Kurshan. Computer-aided Verification of Coordinating Processes: The Automata-Theoretic Approach. Princeton University Press, 1994.

18. B. Li, C. Wang, and F. Somenzi. A satisfiability-based approach to abstraction refinement in model checking. In Workshop on BMC, 2003.

19. J. Marques-Silva and K. Sakallah. GRASP: A search algorithm for propositional satisfiability. IEEETC: IEEE Transactions on Computers, 48, 1999. 
20. K. McMillan. Applying SAT methods in unbounded symbolic model checking. In $C A V, 2003$.

21. K. McMillan. Interpolation and SAT-based model checking. In $C A V, 2003$.

22. K. McMillan and N. Amla. Automatic abstraction without counterexamples. In TACAS, 2003.

23. M. W. Moskewicz, C. F. Madigan, Y. Zhao, L. Zhang, and S. Malik. Chaff: Engineering an Efficient SAT Solver. In $D A C, 2001$.

24. G. Parthasarathy, M. Iyer, K.T. Cheng, and L.C. Wang. A comparison of BDDs, BMC, and sequential SAT for model checking. In High-Level Design Validation and Test Workshop, 2003.

25. M. Prasad, A. Biere, and A. Gupta. A survey of recent advances in sat-based formal verification. In STTT, 2005.

26. J.P. Queille and J. Sifakis. Specification and verification of concurrent systems in CESAR. In Proc. of the 5th International Symposium on Programming, 1982.

27. M. Sheeran, S. Singh, and G. Stalmarck. Checking safety properties using induction and a SAT-solver. In FMCAD, 2000.

28. C. Wang, B. Li, H. Jin, G. Hachtel, and F. Somenzi. Improving ariadne's bundle by following multiple threads in abstraction refinement. In ICCAD, 2003.

29. J. Whittemore, J. Kim, and K. Sakallah. Satire: A new incremental satisfiability engine. In $D A C, 2001$. 